\title{
Knockdown of lncRNA ZFAS1 inhibits progression of nasopharyngeal carcinoma by sponging miR-135a
}

\author{
M. WANG ${ }^{1, *}$, Y. Q. JI ${ }^{1, *}$, Z. B. SONG ${ }^{2}$, X. X. MA ${ }^{3}$, Y. Y. ZOU ${ }^{4}$, X. S. LI ${ }^{1, *}$ \\ ${ }^{1}$ Department of Otorhinolaryngology, Xingtai People's Hospital, Xingtai, Hebei, China; ${ }^{2}$ Department of Internal Medicine, Xingtai County Cen- \\ tral Hospital, Xingtai, Hebei, China; ${ }^{3}$ Department of Internal Medicine, Hebei Eye Hospital, Xingtai, Hebei, China; 4Department of Gynecology, \\ Xingtai People's Hospital, Xingtai, Hebei, China
}

*Correspondence: liymie@163.com

${ }^{*}$ Contributed equally to this work.

Received December 13, 2018 / Accepted May 5, 2019

\begin{abstract}
Nasopharyngeal carcinoma (NPC) is one of the most common head and neck malignancies with leading cause of cancerrelated death. Long noncoding RNAs (lncRNAs) have been reported to play essential roles in progression, prognosis and treatment of patients with NPC. However, the role of lncRNA zinc finger antisense 1 (ZFAS1) and its potential mechanism in NPC progression remain largely unknown. The expression levels of ZFAS1 and microRNA-135a (miR-135a) were measured in NPC tissues and cells by quantitative real-time polymerase chain reaction. The interaction between ZFAS1 and miR-135a was explored by bioinformatics analysis, luciferase reporter assay and RNA immunoprecipitation. Cell proliferation, apoptosis, migration and invasion were analyzed by MTT assay, flow cytometry and Transwell assays, respectively. Our data showed that the expression of ZFAS1 was upregulated and miR-135a was downregulated in NPC tissues and cells. miR-135a was bound to ZFAS1 in NPC cells. Moreover, knockdown of ZFAS1 or overexpression of miR-135a inhibited cell proliferation, migration and invasion but promoted apoptosis in NPC cells. Besides, deficiency of miR-135a reversed silence of ZFAS1-mediated inhibition of NPC progression in vitro. Our data suggested that inhibition of ZFAS1 protected against proliferation, migration and invasion but contributed to apoptosis by sponging miR-135a in NPC cells, providing a novel avenue for NPC treatment.
\end{abstract}

Key words: nasopharyngeal carcinoma, ZFAS1, miR-135a, proliferation, migration, invasion

Nasopharyngeal carcinoma (NPC) is one of the most common head and neck malignancies in southern China with an incidence of 30 per 100,000 persons [1]. With the great advance in the treatment of NPC, many strategies have been used in NPC therapy, such as radiotherapy, chemotherapy and immunotherapy [2]. However, the 5-year survival of NPC patients remains less than 70\% [3]. Hence, it is necessary to explore promising treatment for prognosis and therapeutics of NPC.

Long noncoding RNAs (lncRNAs) have been reported to play important roles in prevention and treatment of NPC by regulating cell proliferation, apoptosis, migration, invasion, drug resistance and angiogenesis [4]. For example, Wu et al. reported that IncRNA urothelial carcinoma-associated 1 facilitates proliferation, migration and invasion in NPC cells [5]. Moreover, $\mathrm{Hu}$ et al. revealed that lncRNA homeobox transcript antisense intergenic RNA promotes cell proliferation, invasion and migration in NPC cells [6]. As for lncRNA zinc finger antisense 1 (ZFAS1), it has been indicated as an oncogene in multiple cancers, contributing to the progression of cancers [7]. In addition, previous studies demonstrated that ZFAS1 could promote development of cancers and is associated with poor prognosis in many cancers, including bladder cancer, non-small-cell lung cancer and gastric cancer [8-10]. Notably, the emerging evidence suggested that ZFAS1 is dysregulated and induces cell proliferation through regulating Wnt/ $\beta$-catenin pathway in NPC [11]. However, the role of ZFAS1 in migration and invasion of NPC and its potential mechanism in NPC progression remain poorly understood.

MicroRNAs (miRNAs), a class of small noncoding RNAs with 18-25 nucleotides, have been reported to play vital roles in progression of human cancers through acting as oncogenes or tumor suppressors [12]. Furthermore, former work suggested miRNAs as therapeutic targets of NPC [13]. Increasing evidences indicated that miR-135a could serve as 
a tumor suppressor in many cancers, such as glioma, breast cancer and gastric cancer [14-16]. Notably, miR-135a is suggested to be lowly expressed in NPC cells [17]. Intriguingly, bioinformatics analysis predicted the potential binding sites of ZFAS1 and miR-135a, stimulating us to pay attention to the interaction between them in NPC. Hence, in this study, we investigated the effect of ZFAS1 on cell proliferation, apoptosis, migration and invasion and explored the relationship between ZFAS1 and miR-135a to elucidate a novel mechanism in NPC progression.

\section{Materials and methods}

Clinical samples. A total of 29 paired cancer tissues and corresponding adjacent normal tissues were collected from patients with NPC from Xingtai People's Hospital. All samples were immediately frozen in liquid nitrogen and then stored at $-80^{\circ} \mathrm{C}$ until used. All participants have signed the informed consent and this study was permitted by the Ethics Committee of Xingtai People's Hospital.

Cell culture and transfection. The human nasopharyngeal epithelial cell line NP69 and NPC cell lines (CNE1, CNE2, HNE1 and HONE1) were purchased from American Tissue Culture Collection (Manassas, VA, USA) and maintained in Dulbecco's Modified Eagle Medium (DMEM; Gibco, CA, USA) containing 10\% fetal bovine serum (FBS; Gibco), 100 $\mathrm{U} / \mathrm{ml}$ penicillin and $100 \mu \mathrm{g} / \mathrm{ml}$ streptomycin (Gibco) at $37^{\circ} \mathrm{C}$ and $5 \% \mathrm{CO}_{2}$.

Small interfering RNA (siRNA) against ZFAS1 (si-ZFAS1), siRNA negative control (si-NC), pcDNA, pcDNA-based ZFAS1 overexpression vector (ZFAS1), miR-135a mimic (miR-135a), mimic negative control (miR-NC), miR-135a inhibitor (anti-miR-135a) and inhibitor negative control (anti-miR-NC) were synthesized by Genepharma (Shanghai, China). Cell transfection was performed in CNE1 and HONE1 cells with $40 \mathrm{nM}$ oligonucleotides or $200 \mathrm{ng}$ vectors by using Lipofectamine 2000 (Invitrogen, Carlsbad, CA, USA) following the manufacturer's instructions. After $24 \mathrm{~h}$ of transfection, cells were collected for further studies.

Quantitative real-time polymerase chain reaction (qRT-PCR). Total RNA was isolated from tissues or cells with TRIzol reagent (Invitrogen) and reverse transcribed using TransScript miRNA First stand cDNA Synthesis SuperMix (TransGen Biotech, Beijing, China), followed by used for qRT-PCR with SYBR green (Applied Biosystems, Foster City, CA, USA) according to the manufacturer's instructions. The qRT-PCR was conducted with the following protocol: $95^{\circ} \mathrm{C}$ for $10 \mathrm{~min}$, and 40 cycles at $95^{\circ} \mathrm{C}$ for $15 \mathrm{~s}$ and $60^{\circ} \mathrm{C}$ for $1 \mathrm{~min}$. The relative expression levels of miR-135a and ZFAS1 were measured with U6 small RNA or $\beta$-actin as internal control using $2^{-\Delta \Delta \mathrm{Ct}}$ method [18]. The primers were used in this study as follows: miR-135a (Forward, 5'-TATGGCTTTTTATTCCTATGTGA-3'; Reverse, 5'-TATGGCTTTTCATTCCTATGTGA-3'), U6 (Forward, 5'-CTCGCTTCGGCAG-
CACA-3'; Reverse, 5'-AACGCTTCACGAATTTGCGT-3'), ZFAS1 (Forward, 5'-CCGGAGTGTGGTACTTCTCC-3'; Reverse, 5'-CCAGAGGTCTCCAACGAAGA-3'), $\beta$-actin (Forward, 5'-CAGCCTTCCTTCTTGGGTAT-3'; Reverse, 5'-TGGCATAGA GGTCTTTACGG-3').

Bioinformatics analysis and luciferase reporter assay. The potential binding sites of miR-135a and ZFAS1 were predicted by bioinformatics analysis using starBase online. To construct the luciferase reporter vector, the sequences of ZFAS1 containing the putative binding sites of miR-135a were amplified and inserted in pGL3-promoter reporter vector (Promega, Madison, WI, USA). Corresponding mutant reporter vector was obtained by mutating the binding sites (AAGCCAU) to (GAAUAGC). CNE1 and HONE1 cells were co-transfected with wild-type (WT) or mutant (MUT) luciferase reporter vectors (ZFAS1-WT or ZFAS1-MUT), and miR-135a mimic or miR-NC using Lipofectamine ${ }^{\mathrm{TM}} 2000$ according to the manufacturer's protocols. After the transfection for $48 \mathrm{~h}$, cells were collected for luciferase activity assay using luciferase reporter assay kit (Promega) according to the manufacturer's instructions.

RNA immunoprecipitation (RIP). RIP assay was performed by using Magna RNA immunoprecipitation kit (Millipore, MA, USA) according to the manufacturer's protocols. In brief, CNE1 and HONE1 cells transfected with miR-135a mimic or miR-NC were lysed and incubated in RIP immunoprecipitation buffer containing magnetic beads bound with antibody against Ago 2 or IgG. The level of ZFAS1 enriched on beads were analyzed by qRT-PCR.

Cell proliferation. 3-(4,5-dimethyl-2-thiazolyl)-2,5diphenyl-2-H-tetrazolium bromide (MTT) assay was used to measure cell proliferation. CNE1 and HONE1 cells were seeded into 96-well plates at a density of 5,000 cells per well. After the culture for $0,24,48$ or $72 \mathrm{~h}$, cells were incubated with $0.5 \mathrm{mg} / \mathrm{ml}$ MTT solution (Sigma, MO, USA) for another $4 \mathrm{~h}$, followed by interacted with $100 \mu \mathrm{l}$ of dimethylsulfoxide (Thermo Fisher, DE, USA). Following the solubilization of formazan, the absorbance was measured at $490 \mathrm{~nm}$ using a microplate reader (Bio-Rad, CA, USA).

Cell apoptosis. Annexin V-FITC/PI apoptosis detection kit (Yeasen, Shanghai, China) was used for measurement of cell apoptosis by flow cytometry. After the culture for $72 \mathrm{~h}$, CNE1 and HONE1 cells were resuspended in binding buffer, and then stained with $5 \mu \mathrm{l}$ Annexin V-FITC and $10 \mu \mathrm{l}$ PI for $10 \mathrm{~min}$ in the dark. The apoptotic cells were examined via using a flow cytometer (Becton Dickinson, CA, USA).

Transwell assay was performed to measure cell migration and invasion using transwell chambers (Corning, NY, USA). In brief, CNE1 and HONE1 cells $\left(2 \times 10^{4}\right.$ cells/well) in serumfree DMEM medium were seeded in the upper chambers, and $600 \mu \mathrm{l}$ DMEM medium containing 10\% FBS was placed in the lower chambers. After the incubation for $24 \mathrm{~h}$, migrated cells through the membranes were stained with $0.1 \%$ crystal violet (Sigma) and then observed under a $\times 200$ magnification microscope (Olympus, Tokyo, Japan) in five random 
fields. For the invasion assay, the transwell chambers were pre-coated with Matrigel (Becton Dickinson) and experiment was performed following the same approach.

Statistical analysis. Data were presented as the mean \pm standard deviation (S.D.) from three independent experiments. The differences between groups were analyzed by student's t-test or one-way ANOVA using GraphPad Prism 7 (GraphPad Inc., CA, USA). A p-value $<0.05$ was regarded as statistical significance.

\section{Results}

ZFAS1 is highly expressed in NPC. To explore the potential role of ZFAS1 in NPC progression, its expression was measured in NPC tissues and cells. qRT-PCR assay showed that the expression of ZFAS1 was abnormally elevated in NPC tissues compared with that in adjacent normal samples $(n=29)$ (Figure 1A). Moreover, the abundance of ZFAS1 was much higher in NPC cells (CNE1, CNE2, HNE1 and HONE1) than in control NP69 cells (Figure 1B). Consequently, CNE1 and HONE1 cells with relative higher expression of ZFAS1 were used for subsequent experiments.

ZFAS1 is a sponge of miR-135a. To explore the mechanism that allows ZFAS1 in NPC progression, promising miRNAs bound to ZFAS1 were explored by bioinformatics analysis using starBase, which predicted the putative binding sites of ZFAS1 and miR-135a. To validate this prediction, wild-type or mutant luciferase reporter vectors ZFAS1-WT or ZFAS1-MUT were generated and transfected into CNE1 and HONE1 cells. (Figure 2A). As shown in Figures 2B-2D, the abundance of miR-135a was effectively enhanced in CNE1 and HONE1 cells by transfection of miR-135a mimic compared with that in miR-NC group. Moreover, upregulation of miR-135a led to a great loss of luciferase activity in CNE1 and HONE1 cells transfected with ZFAS1-WT, while its efficacy was lost in response to ZFAS1-MUT group.
A

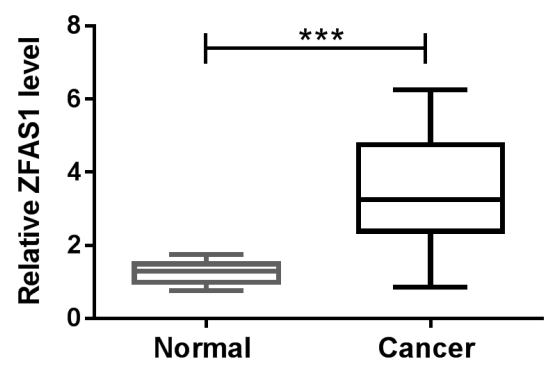

B

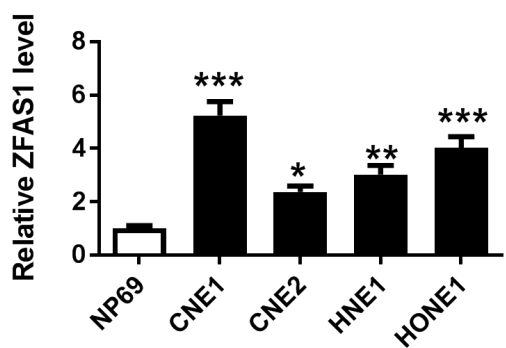

Figure 1. ZFAS1 expression is enhanced in NPC tissues and cells. A) The expression of ZFAS1 was measured in NPC tissues and adjacent normal samples by qRT-PCR. $\mathrm{n}=29$. B) The abundance of ZFAS1 was detected in NPC cells and control cells by $\mathrm{qRT}-\mathrm{PCR}$. ${ }^{\star} \mathrm{p}<0.05,{ }^{* *} \mathrm{p}<0.01,{ }^{\star * *} \mathrm{p}<0.001$.

A Site: $c h r 20: 47895149-47895174[+]$

ZFAS1-WT 5'... auauaagggagguucaggAAGCC AUu ....3' miR-135a 3'aguguauccuuauuuUUCGGUAu 5'

ZFAS1-MUT 5'... auauaagggagguucaggGAAUAGCu ...3'

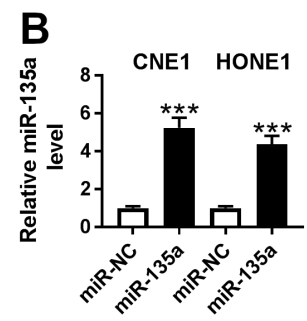

C

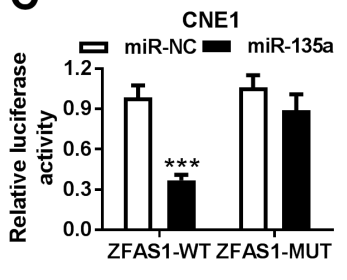

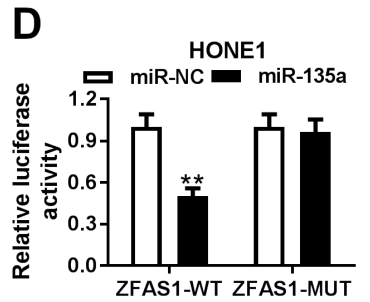
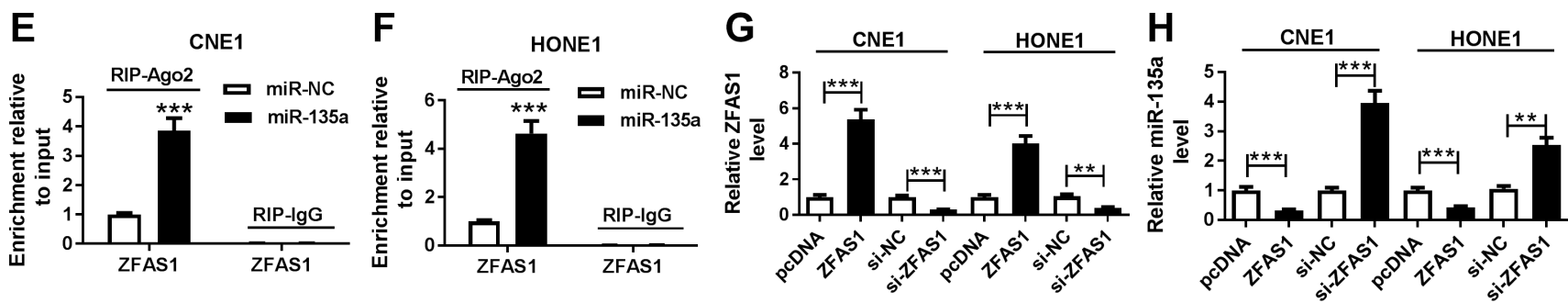

Figure 2. miR-135a is sponged by ZFAS1. A) The putative binding sites of ZFAS1 and miR-135a were predicted by starBase. B) The expression of miR-135a was measured in CNE1 and HONE1 cells transfected with miR-135a mimic or mimic negative control (miR-NC) by $\mathrm{qRT}$-PCR. C and D) Luciferase activity was detected in CNE1 and HONE1 cells co-transfected with luciferase reporter vectors ZFAS1-WT or ZFAS1-MUT and miR-NC or miR-135a. E and F) The level of ZFAS1 enriched by Ago2 RIP was measured in CNE1 and HONE1 cells transfected with miR-135a or miR-NC by qRT-PCR. G and H) The expression levels of ZFAS1 and miR-135a were detected in CNE1 and HONE1 cells transfected with pcDNA, ZFAS1, si-NC or si-ZFAS1 by qRT-PCR. ${ }^{* *} \mathrm{p}<0.01,{ }^{* *} \mathrm{p}<0.001$. 
Furthermore, overexpression of miR-135a resulted in higher level of ZFAS1 enriched by Ago2 RIP, whereas it failed to show efficacy of enrichment in IgG RIP group (Figures 2E and $2 \mathrm{~F}$ ). In addition, the effect of ZFAS1 on miR-135a expression was investigated in CNE1 and HONE1 cells through overexpressing ZFAS1 or knocking down ZFAS1, which was confirmed in Figure 2G. The data of qRT-PCR also revealed that the level of miR-135a was notably decreased by overexpression of ZFAS1 and increased by knockdown of ZFAS1 in CNE1 and HONE1 cells (Figure 2H).

miR-135a is lowly expressed in NPC. Seeing that miR-135a is bound to ZFAS1, the expression of miR-135a was also detected in NPC. Compared with that in normal samples, miR-135a level was obviously reduced in NPC cancer tissues $(n=29)$ (Figure 3A). Furthermore, the abundance of miR-135a was significantly decreased in NPC cells (CNE1, CNE2, HNE1 and HONE1) compared with that in control NP69 cells (Figure 3B).

Depletion of ZFAS1 or miR-135a addition inhibits progression of NPC. To explore the roles of ZFAS1 and miR-135a in NPC progression, CNE1 and HONE1 cells were transfected with si-NC, si-ZFAS1, miR-NC or miR-135a. After the transfection, cell proliferation was obviously reduced in CNE1 and HONE1 cells by silencing ZFAS1 at 48 and $72 \mathrm{~h}$ (Figure 4A). Similarly, overexpression of miR-135a significantly decreased the proliferation rate of CNE1 and HONE1 cells at 48 and $72 \mathrm{~h}$ (Figure 4B). Moreover, knockdown of ZFAS1 or addition of miR-135a led to great production of apoptosis in CNE1 and HONE1 cells (Figures 4C and 4D). In addition, knockdown of ZFAS1 strikingly limited
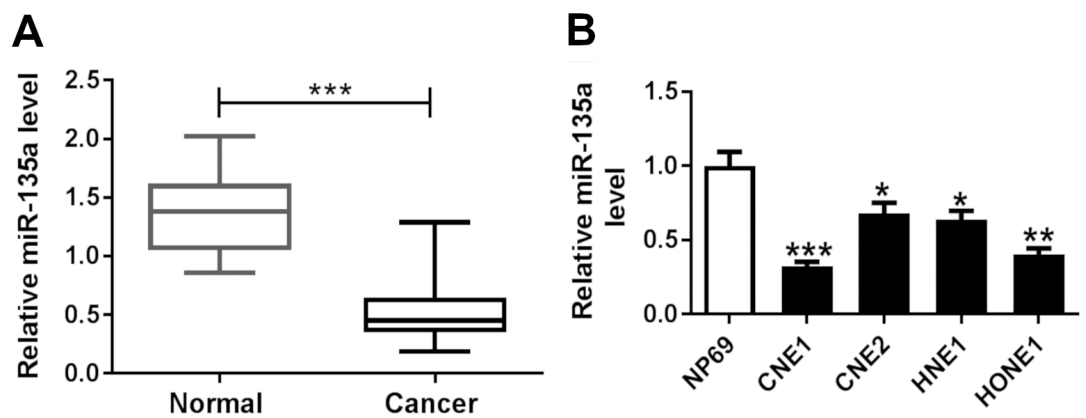

Figure 3. miR-135a expression is reduced in NPC tissues (A) and cell lines (B). The expression of miR-135a in NPC tissues and cells was measured by qRT-PCR. ${ }^{*} \mathrm{p}<0.05,{ }^{* *} \mathrm{p}<0.01,{ }^{* * *} \mathrm{p}<0.001$.

A
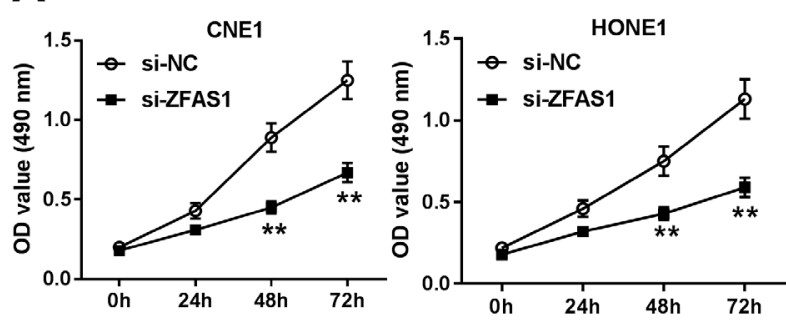

C
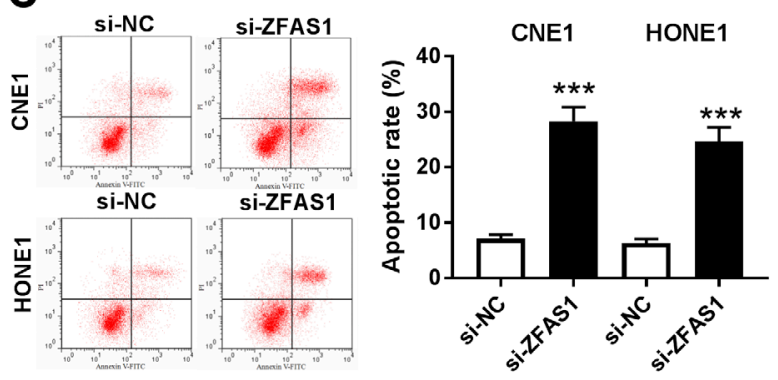

B
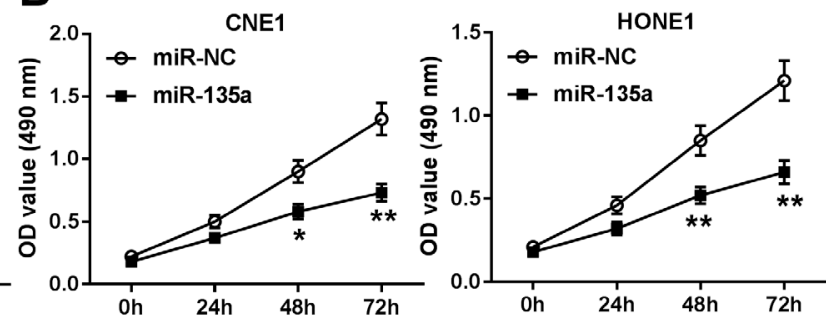

D

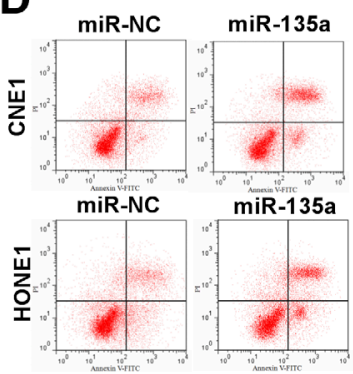

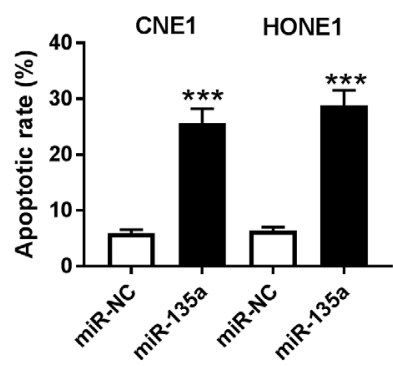

Figure 4. ZFAS1 knockdown or miR-135a overexpression inhibits proliferation and promotes apoptosis in NPC cells. A and C) Cell proliferation and apoptosis were measured in CNE1 and HONE1 cells transfected with si-NC or si-ZFAS1 by MTT assay and flow cytometry, respectively. B and D) Cell proliferation and apoptosis were detected in CNE1 and HONE1 cells transfected with miR-NC or miR-135a by MTT assay and flow cytometry, respectively. ${ }^{*} \mathrm{p}<0.05,{ }^{* *} \mathrm{p}<0.01,{ }^{* *} \mathrm{p}<0.001$. 
the migratory and invasive abilities of CNE1 and HONE1 cells (Figures 5A and 5B). The results of Transwell assay also demonstrated that overexpression of miR-135a resulted in obvious suppression of migration and invasion in CNE1 and HONE1 cells (Figures 5C and 5D).

Deficiency of miR-135a attenuates interference of ZFAS1-mediated NPC progression inhibition. To investigate whether miR-135a is required for the regulatory mechanism of ZFAS1 in NPC progression, CNE1 and HONE1 cells were transfected with si-NC, si-ZFAS1, si-ZFAS1 and antimiR-NC or anti-miR-135a. After the transfection, knockdown of miR-135a significantly alleviated the inhibitory effect of ZFAS1 interference on cell proliferation in CNE1 and HONE1 cells (Figures 6A and 6B). Moreover, inhibition of miR-135a markedly abated silence of ZFAS1-induced apoptosis in CNE1 and HONE1 cells (Figures 6C and 6D). Besides, knockdown of miR-135a rescued the migratory and invasive abilities of CNE1 and HONE1 cells from ZFAS1 knockdown (Figures 6E-6H).

\section{Discussion}

Previous study has reported lncRNAs as potential therapeutic targets for NPC by addressing metastasis [19]. ZFAS1, as an oncogenic lncRNA, has been indicated as unfavorable prognostic factor and therapeutic target for treatment of multiple cancers $[20,21]$. However, the function of ZFAS1 is largely unknown in NPC. Therefore, we investigated the effect of ZFAS1 on migration and invasion in NPC cells and explored the underlying mechanism in progression of NPC. This study first demonstrated that inhibition of ZFAS1 could upregulate miR-135a expression to inhibit cell proliferation, migration and invasion but to induce apoptosis in NPC cells.

In our study, we found that ZFAS1 expression was increased in NPC tissues and cells, suggesting its potential carcinogenic role in NPC. Moreover, knockdown of ZFAS1 inhibited proliferation and increased apoptosis in NPC cells, which is also in agreement with previous study [11]. Besides, we first demonstrated that interference of ZFAS1 blocked the abilities of migration and invasion in NPC cells, which is also consistent with the reports that ZFAS1 contributed to cell migration and invasion in other cancers $[22,23]$. These findings revealed that ZFAS1 functioned as an oncogene in NPC progression. However, the potential mechanism that allows ZFAS1 participating in NPC progression remains poorly understood. Former works indicated that ZFAS1 could act as miRNA sponge to regulate the target expressions in many conditions. For example, ZFAS1 promoted migration and invasion in rheumatoid arthritis by sponging miR-27a [24]. Moreover, silencing ZFAS1 suppressed cell proliferation and promoted apoptosis by regulating miR-329 in bladder cancer [25]. Additionally, knockdown of ZFAS1 protected against acute myocardial infarction by mediating miR-150 and C-reactive protein [26]. Therefore, to figure out whether ZFAS1 serves as a miRNA sponge in NPC, the potential miRNA was explored by bioinformatics analysis
A
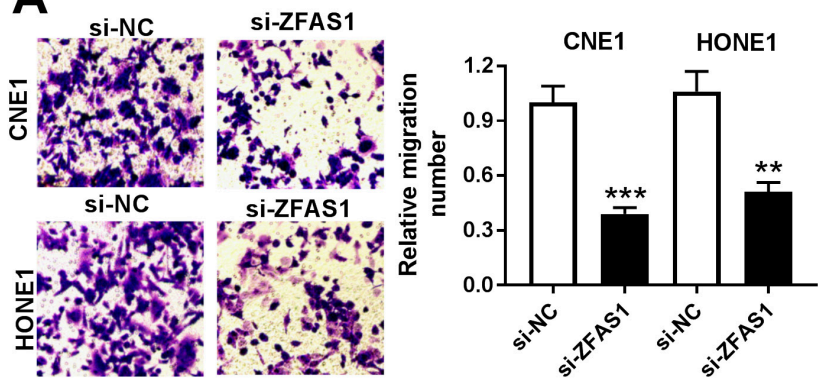

C
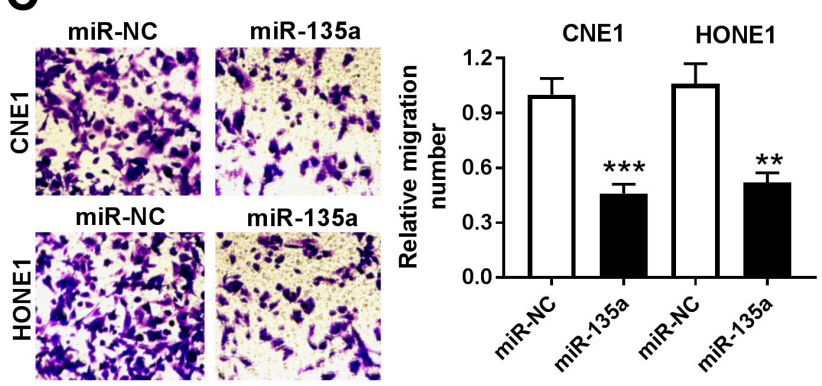

B
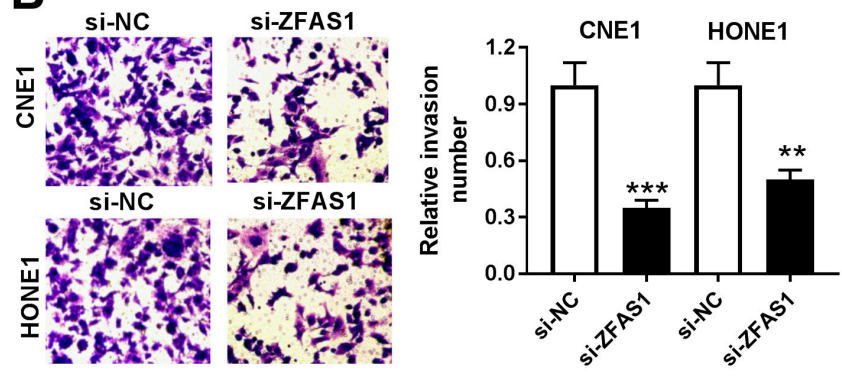

D
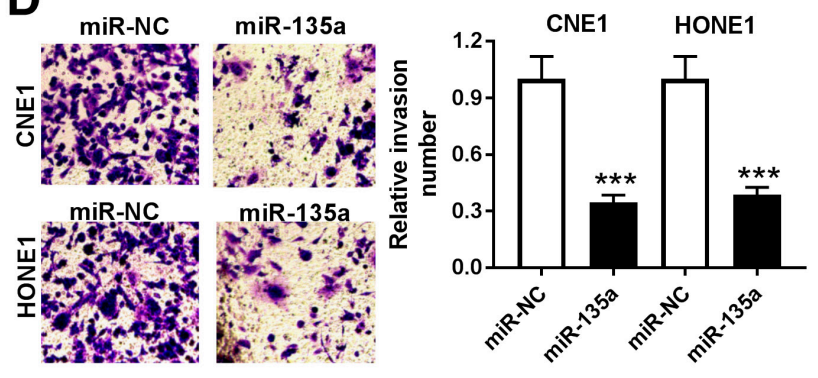

Figure 5. ZFAS1 inhibition or miR-135a overexpression suppresses migration and invasion in NPC cells. A and B) Cell migration and invasion were measured in CNE1 and HONE1 cells transfected with si-NC or si-ZFAS1 by Transwell assay. C and D) Cell migration and invasion were detected in CNE1 and HONE1 cells transfected with miR-NC or miR-135a by transwell assay. ${ }^{* *} \mathrm{p}<0.01,{ }^{* * *} \mathrm{p}<0.001$. 

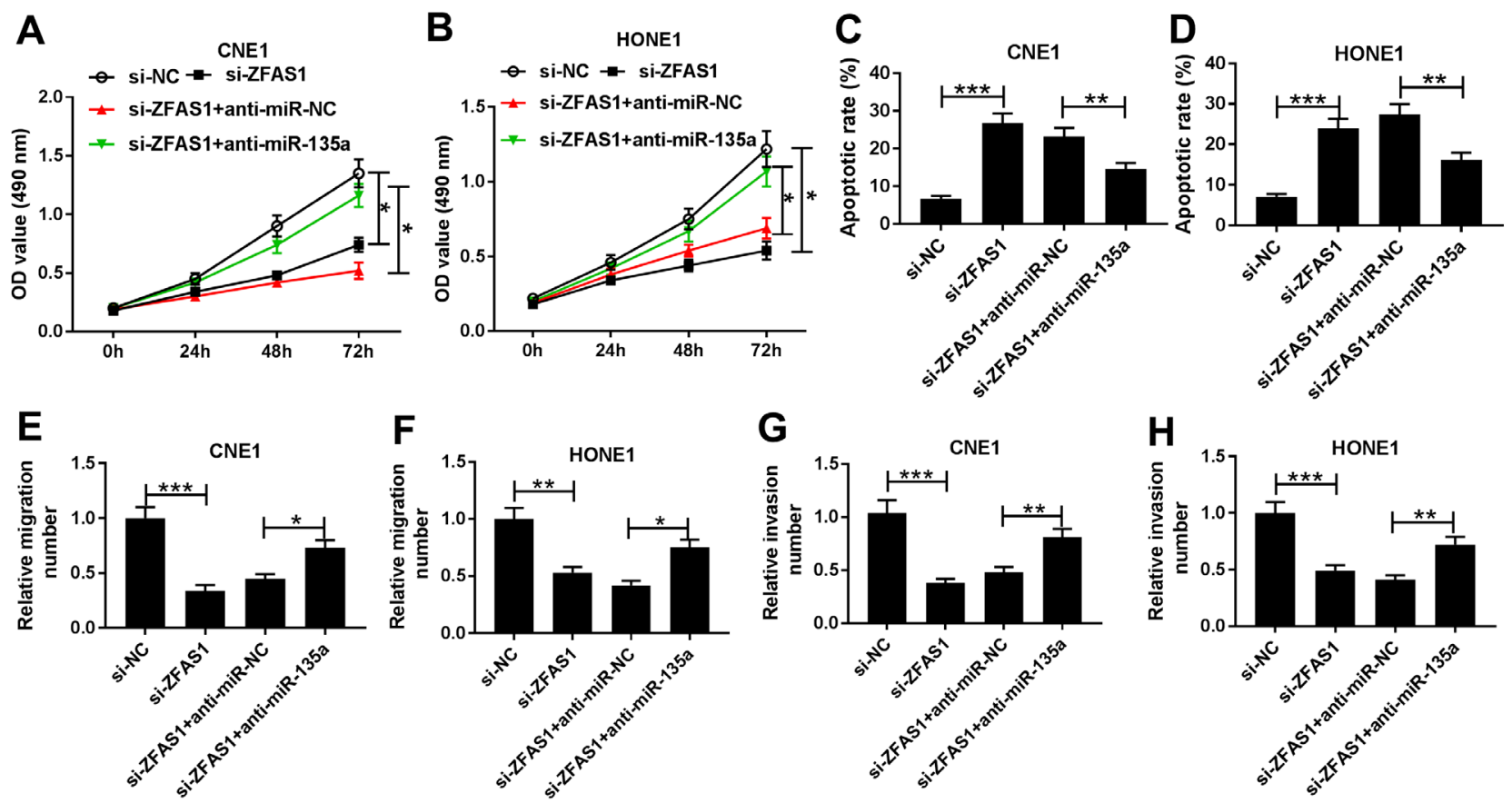

Figure 6. Abrogation of miR-135a reverses interference of ZFAS1-mediated NPC progression inhibition. Cell proliferation (A and B), apoptosis (C and D), migration (E and F) and invasion ( $G$ and $H)$ were measured in CNE1 and HONE1 cells transfected with si-NC, si-ZFAS1, si-ZFAS1 and anti-miRNC or anti-miR-135a by MTT assay, flow cytometry and transwell assay, respectively. ${ }^{*} \mathbf{p}<0.05,{ }^{* *} \mathbf{p}<0.01,{ }^{* * *} \mathrm{p}<0.001$.

and results showed miR-135a containing the binding sites of ZFAS1. Subsequently, the interaction between ZFAS1 and miR-135a was validated by luciferase reporter assay and RIP assay.

Previous studies have reported that miR-135a acted as a tumor suppressor in human cancers. For instance, miR-135a inhibited proliferation and migration by regulating $\mathrm{Na} / \mathrm{H}$ Exchanger isoform 9 in glioblastoma cells [27]. Furthermore, Zhang et al. reported that miR-135a suppressed cell growth and metastasis in pancreatic cancer [28]. In addition, Shi et al. revealed that miR-135a inhibited migration, invasion and epithelial-mesenchymal transition by targeting krüppel-like factor 8 in lung cancer [29]. What's more, former effort showed that miR-135a was downregulated in NPC cells and it played an anti-cancer role in NPC [17]. Similarly, in this study we also showed low expression of miR-135a in NPC cells and its addition inhibited NPC progression. Notably, miR-135a absence attenuated knockdown of ZFAS1-mediated therapeutic effect on NPC in vitro. Thus, we provided insight into ZFAS1 regulating malignancy of NPC by sponging miR-135a.

However, there were some limitations in the present study. First, the sample size should be increased to further investigate the effect of ZFAS1 on prognosis of NPC patients. Then the pre-clinical experiments should be performed to analyze the regulatory role of ZFAS1 in vivo by xenograft model in future. Furthermore, the promising targets of miR-135a and signaling pathway should be explored for better understanding the mechanism in further study.

In conclusion, ZFAS1 was highly expressed and miR-135a was lowly expressed in NPC tissues and cells. Silencing ZFAS1 repressed proliferation, migration and invasion but triggered apoptosis in NPC cells, possibly by sponging miR-135a, providing a novel mechanism for progression and pathogenesis of NPC and indicating a new potential strategy for treatment of NPC.

\section{References}

[1] LEE A, MA B, NG W, CHAN A. Management of Nasopharyngeal Carcinoma: Current Practice and Future Perspective. J Clin Oncol 2015; 33: 3356-3364. https://doi.org/10.1200/ JCO.2015.60.9347

[2] CHUA M, WEE J, HUI E, CHAN A. Nasopharyngeal carcinoma. Lancet 2016; 387: 1012-1024. https://doi.org/10.1016/ S0140-6736(15)00055-0

[3] GENOVA P, BRUNETTI F, BEQUIGNON E, LANDI F, LIZZI $\mathrm{V}$ et al. Solitary splenic metastasis from nasopharyngeal carcinoma: a case report and systematic review of the literature. World J Surg Oncol 2016; 14: 184. https://doi. org/10.1186/s12957-016-0941-2

[4] WU J, HANN S. Functions and Roles of Long-NonCoding RNAs in Human Nasopharyngeal Carcinoma. Cell Physiol Biochem 2018; 45: 1191-1204. https://doi. org/10.1159/000487451 
[5] WU J, DU M, ZHANG Q, ZHANG W, FAN Y et al. Long noncoding RNA UCA1 promotes the proliferation, invasion, and migration of nasopharyngeal carcinoma cells via modulation of miR-145. Onco Targets Ther 2018; 11: 7483-7492. https://doi.org/10.2147/OTT.S182290

[6] HU W, XU W, SHI Y, DAI W. IncRNA HOTAIR upregulates COX-2 expression to promote invasion and migration of nasopharyngeal carcinoma by interacting with miR-101. Biochem Biophys Res Commun 2018; 505: 1090-1096. https:// doi.org/10.1016/j.bbrc.2018.09.19

[7] HE A, HE S, LI X, ZHOU L. ZFAS1: A novel vital oncogenic lncRNA in multiple human cancers. Cell Prolif 2019; 52: e12513. https://doi.org/10.1111/cpr.12513

[8] YANG H, LI G, CHENG B, JIANG R. ZFAS1 functions as an oncogenic long non-coding RNA in bladder cancer. Biosci Rep 2018; 38. https://doi.org/10.1042/BSR20180475

[9] TIAN F, MENG F, WANG X. Overexpression of long-noncoding RNA ZFAS1 decreases survival in human NSCLC patients. Eur Rev Med Pharmacol Sci 2016; 20: 5126-5131.

[10] PAN L, LIANG W, FU M, HUANG Z, LI X et al. Exosomesmediated transfer of long noncoding RNA ZFAS1 promotes gastric cancer progression. J Cancer Res Clin Oncol 2017; 143: 991-1004. https://doi.org/10.1007/s00432-017-2361-2

[11] CHEN X, LI J, LI C, LU X. Long non-coding RAN ZFAS1 promotes nasopharyngeal carcinoma through activation of Wnt/ $\beta$-catenin pathway. Eur Rev Med Pharmacol Sci 2018; 22: 3423-3429. https://doi.org/10.26355/eurrev_201806_15165

[12] KWAN J, PSARIANOS P, BRUCE J, YIP K, LIU F. The complexity of microRNAs in human cancer. J Radiat Res 2016; 57: i106-111. https://doi.org/10.1093/jrr/rrw009

[13] LEE K, TAN J, LAM A, GAN S. MicroRNAs serving as potential biomarkers and therapeutic targets in nasopharyngeal carcinoma: A critical review. Crit Rev Oncol Hematol 2016; 103: 1-9. https://doi.org/10.1016/j.critrevonc.2016.04.006

[14] SHI H, WANG D, XU F, TENG J, WANG Y. miR-135a inhibits glioma cell proliferation and invasion by directly targeting FOXO1. Eur Rev Med Pharmacol Sci 2018; 22: 4215-4223. https://doi.org/10.26355/eurrev_201807_15415

[15] AHMAD A, ZHANG W, WU M, TAN S, ZHU T. Tumorsuppressive miRNA-135a inhibits breast cancer cell proliferation by targeting ELK1 and ELK3 oncogenes. Genes Genomics 2018; 40: 243-251. https://doi.org/10.1007/ s13258-017-0624-6

[16] ZHANG C, CHEN X, CHEN X, WANG X, JI A et al. miR$135 \mathrm{a}$ acts as a tumor suppressor in gastric cancer in part by targeting KIFC1. Onco Targets Ther 2016; 9: 3555-3563. https://doi.org/10.2147/OTT.S105736

[17] WANG L, KANG Z, YANG Z, MA R, TAN Y et al. MicroRNA-135a Inhibits Nasopharyngeal Carcinoma Cell Proliferation Through Targeting Interleukin-17. Cell Physiol Biochem 2018; 46: 2232-2238. https://doi.org/10.1159/000489591
[18] LIVAK KJ, SCHMITTGEN TD. Analysis of relative gene expression data using real-time quantitative PCR and the 2- $\Delta \Delta$ C T Method. Methods 2001; 25: 402-408. https://doi. org/10.1006/meth.2001.1262

[19] WEN X, TANG X, LI Y, REN X, HE Q et al. Microarray Expression Profiling of Long Non-Coding RNAs Involved in Nasopharyngeal Carcinoma Metastasis. Int J Mol Sci 2016; 17: E1956. https://doi.org/10.3390/ijms17111956

[20] GAO K, JI Z, SHE K, YANG Q, SHAO L. Long non-coding RNA ZFAS1 is an unfavourable prognostic factor and promotes glioma cell progression by activation of the Notch signaling pathway. Biomed Pharmacother 2017; 87: 555-560. https://doi.org/10.1016/j.biopha.2017.01.014

[21] XU W, HE L, LI Y, TAN Y, ZHANG F et al. Silencing of lncRNA ZFAS1 inhibits malignancies by blocking Wnt/ $\beta$ catenin signaling in gastric cancer cells. Biosci Biotechnol Biochem 2018; 82: 456-465. https://doi.org/10.1080/091684 51.2018.1431518

[22] LI T, XIE J, SHEN C, CHENG D, SHI Y et al. Amplification of Long Noncoding RNA ZFAS1 Promotes Metastasis in Hepatocellular Carcinoma. Cancer Res 2015; 75: 3181-3191. https://doi.org/10.1158/0008-5472.CAN-14-3721

[23] WANG W, XING C. Upregulation of long noncoding RNA ZFAS1 predicts poor prognosis and prompts invasion and metastasis in colorectal cancer. Pathol Res Pract 2016; 212: 690-695. https://doi.org/10.1016/j.prp.2016.05.003

[24] YE Y, GAO X, YANG N. LncRNA ZFAS1 promotes cell migration and invasion of fibroblast-like synoviocytes by suppression of miR-27a in rheumatoid arthritis. Hum Cell 2018; 31: 14-21. https://doi.org/10.1007/s13577-017-0179-5

[25] WANG J, LIU Q, CHENG X, ZHANG W, JIN Y. The long noncoding RNA ZFAS1 facilitates bladder cancer tumorigenesis by sponging miR-329. Biomed Pharmacother 2018; 103: 174-181. https://doi.org/10.1016/j.biopha.2018.04.031

[26] WU T, WU D, WU Q, ZOU B, HUANG X et al. Knockdown of Long Non-Coding RNA-ZFAS1 Protects Cardiomyocytes Against Acute Myocardial Infarction Via Anti-Apoptosis by Regulating miR-150/CRP. J Cell Biochem 2017; 118: 32813289. https://doi.org/10.1002/jcb.25979

[27] GOMEZ ZUBIETA D, HAMOOD M, BEYDOUN R, PALL A, KONDAPALLI K. MicroRNA-135a regulates NHE9 to inhibit proliferation and migration of glioblastoma cells. Cell Commun Signal 2017; 15: 55. https://doi.org/10.1186/ s12964-017-0209-7

[28] ZHANG X, GAO F, ZHOU L, WANG H, SHI G et al. UCA1 Regulates the Growth and Metastasis of Pancreatic Cancer by Sponging miR-135a. Oncol Res 2017; 25: 1529-1541. https://doi.org/10.3727/096504017X14888987683152

[29] SHI H, JI Y, ZHANG D, LIU Y, FANG P. MiR-135a inhibits migration and invasion and regulates EMT-related marker genes by targeting KLF8 in lung cancer cells. Biochem Biophys Res Commun 2015; 465: 125-130. https://doi. org/10.1016/j.bbrc.2015.07.145 